International Journal on Artificial Intelligence Tools

Vol. 18, No. 6 (2009) 949-957

(C) World Scientific Publishing Company

\title{
A NEW PARAMETERIZED POTENTIAL FAMILY FOR PATH PLANNING ALGORITHMS
}

\author{
FABRICIO FERRARI \\ Universidade Federal do Pampa, Bagé, RS, Brazil \\ fabricio.ferrari@unipampa.edu.br
}

Received 15 April 2008

Accepted 18 April 2009

\begin{abstract}
In this work, it is proposed a new family of potentials for path planning algorithms, one kind to the goal and other to the obstacles. With these new potentials it is possible to parameterize the potential scale length and strength easily, providing better control over the moving object path characteristics. In this way, the path problem can be treated analytically. For example, the minimum distance between the moving object and the obstacles can be calculated as a function of the potential parameters. Simulations are made to test its ability to guide a vehicle through an obstacle-free path towards the goal. The success rate of the moving object on reaching the goal is compared with the potential parameters and with obstacle configuration and distribution parameters.
\end{abstract}

Keywords: Autonomous navigation; potential theory; path planning.

\section{Introduction}

In many situations, it may be necessary to guide a moving object (MO) to a definite configuration in its degrees of freedom. In these situations, the movements may be done while avoiding certain positions where the MO collide with other objects in space, the obstacles. This is the case of a vehicle in a street, a robot arm in an industry or the task of moving a piano out of a room. In these situations, a valuable virtue an autonomous MO may have is the ability to guide itself to a defined goal while avoiding the obstacles. Several techniques, called path planning algorithms, claim to solve this problem by using, for example, road map methods, cell decomposition or potential field methods. ${ }^{1}$ Potential field methods, such as the one presented here, are important because of its formal simplicity and low computational cost and low complexity.

\section{The Potential Field Method}

The potential field method ${ }^{1-4}$ consists in assigning to each obstacle and goal, a repulsive and attractive virtual potential, respectively. In this way, virtual forces will cause the MO to be repelled by the obstacles and attracted by the goal. Po- 
tential field method have the advantage of not being computationally expensive, being possible to calculate a path between hundreds of obstacles even with simple processors, for example those found in embedded systems. Further, its expansion to higher dimensional spaces is analytically simple and the increase in computation raises linearly with the space dimension. With other methods, e.g. road map, Cspace or cell decomposition, computation raises as a power of the space dimension and the need of memory is many times greater.

Besides its virtues, one limitation of potential field methods are local minima, points where the potential are constant and hence the force is zero. In these points, the MO is trapped and the movement ceases although the goal has not been reached. So these algorithms are not complete in the sense that it is not guaranteed that they find a path to the goal, if it exists. Anyway, there are various techniques that attempt to overcome these limitations, for example by using harmonic functions in the potential, ${ }^{5}$ by using a global path planner or heuristics. ${ }^{6}$ Most methods that saves the MO from these local minimum trap in general can be successfully adapted to the method presented in this work. Although this is critical, here we will be concerned with the idiosyncrasies of the current potential family; we shall address the specific problem of local minima in future work.

Another important issue regarding potential field methods is the situation of goals non-reachable with obstacles nearby (GNRON), as explained in Ref. 7. In this case, obstacles too close to the goal do not allow the MO to reach it, a local minimum. With the potentials used in this work, it may be possible to avoid this situation by choosing adequate potential parameters to limit the obstacle potential range.

The method can also be used in conjunction with other methods, leaving the large scale planning to the potential field algorithm and using an alternative method for local planning or in the case of local minimum. Also, in order to apply it to dynamical environments the algorithm can consider it as a series of static ones, at each time step the algorithm recalculates the potential field based on the new positions.

Several authors have studied different potential functions for path planning, such as Newtonian potentials ${ }^{8}$ and general inverse distance potentials, ${ }^{1,3}$ for example. In this work, the idea of controlling the path characteristics by means of the potentials parameters is introduced, being this parameters the potential scale length and degree. The purpose of this work is to explore the analytical formalism behind this scenario, providing theoretical tools to investigate and use potential fields in path planning.

\section{The Potentials}

Consider a workspace where a point MO, the goal and the $N$ obstacles are located. In this workspace, the center position of the MO is indicated by the vector $\mathbf{q}$, of the goal by $\mathbf{q}_{g}$ and of the $N$ obstacles by the set $\left\{\mathbf{q}_{i}\right\}, i=1 \ldots N$. We may write a 
vector which points from the MO to the goal and to the obstacles, respectively,

$$
\mathbf{r}_{g} \equiv \mathbf{q}_{g}-\mathbf{q} \quad \text { and } \quad \mathbf{r}_{i} \equiv \mathbf{q}_{i}-\mathbf{q}, \quad i=1 \ldots N .
$$

The magnitudes of these vectors, $r_{g}$ and $\left\{r_{i}\right\}$, are the relative distances in question. The vectors $\hat{\mathbf{e}}_{g}$ and $\left\{\hat{\mathbf{e}}_{i}\right\}$, are the normalized versions of $\mathbf{r}_{g}$ and $\left\{\mathbf{r}_{i}\right\}$, respectively. We may now define the obstacles and goal potentials in terms of these quantities.

Different potential functions have been used with path planning algorithms, for example those proposed by Refs. 3 and 1 , which consider an attractive $\psi$ and a repulsive $\varphi$ potentials

$$
\psi\left(r_{g}\right)=A r_{g}^{m}, \quad \varphi\left(r_{i}\right)=B\left(\frac{1}{r_{i}}-\frac{1}{a}\right)
$$

valid for $r_{i}<a$ and considered 0 for $r_{i}>a$. The parameters $A$ and $B$ are the potential intensities, $a$ is the scale length where the repulsion ceases and $m$ the attractive potential degree, almost invariably set to 1 or 2 . A modified version of these potentials were used by Ref. 7 to solve the GNRON problem, but the potentials resembles the originals in its dependence with the distance. More recently, ${ }^{9}$ introduced anisotropic potentials with angle varying magnitudes to try to solve the problem of local minimum (see Section 2). Their potentials angular distribution can be adapted to the current potentials. The advantage of the new potentials introduced in this work is to allow the control of the overall MO path characteristics by changing its parameters. It's worth noting that the formalism developed here does not depend on the potential functional form, although the specific result does.

\subsection{The obstacles potential}

Suppose the obstacle repulsive potential $\varphi\left(r_{i}\right)$ to be of exponential form, with the power of the distance as argument. Its range is adjustable by means of a scale length $a$ and a gradient degree $n$. We may also consider it normalized at $r_{i}=a$. Then

$$
\varphi\left(r_{i}\right)=\exp \left[1-\left(\frac{r_{i}}{a}\right)^{n}\right] .
$$

Being so, the vector force, defined as $\mathbf{F}_{\varphi}(r)=-\nabla \varphi(r)$, is

$$
\mathbf{F}_{\varphi}\left(r_{i}\right)=-\frac{n}{a}\left(\frac{r_{i}}{a}\right)^{n-1} \exp \left[1-\left(\frac{r_{i}}{a}\right)^{n}\right] \hat{\mathbf{e}}_{i} .
$$

The potential and force profiles are shown for different $n$ in Figure 1. The minus sign explicits the fact the force direction at the MO is opposite to the obstacle location. At a distance $r_{i}=a$ from the obstacle the force is $\mathbf{F}_{\varphi}(a)=-(n / a) \hat{\mathbf{e}}_{i}$, so the characteristic size and effect of the potential and force of each obstacle can be controlled by the parameters $a$ and $n$, respectively (see Section 4). The radius where the force is maximum, $r_{\max }$, is given by $r_{\max }=a[1-(1 / n)]^{1 / n}$, with $\lim _{n \rightarrow \infty} r_{\max }=a$. The maximum force which may be experienced at this point is then $\mathbf{F}_{\varphi}\left(r_{\max }\right)=(1-n)\left(e^{1 / n} / r_{\max }\right) \hat{\mathbf{e}}_{i}$. 

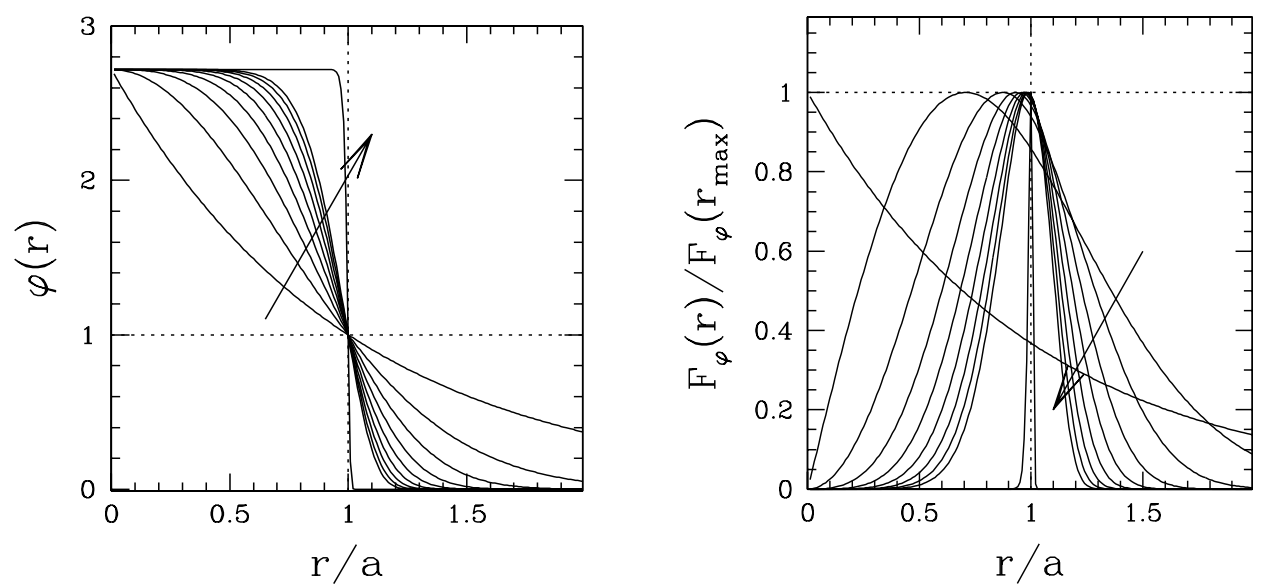

Fig. 1. Obstacles Exponential Family: potential $\varphi$ (top) and force $F_{\varphi}$ (bottom) according to Eq. (2) and Eq. (3) for $n=1-9$ and $100 ; n$ raises in the direction indicated by the arrows.

It should be noted that the force is not infinite as the distance approaches zero. This may be considered a weakness of the potential, for once the MO passes the region of maximum force, it will collide with the obstacle. But, since the force strength is big enough to avoid it, which can be guaranteed by suitably choosing $n$ and $a$, there is no problem in a bounded value force. This argument is reinforced by the results of simulations in Section 5 , where no collision occurred, the MO were only trapped in local minima.

\subsection{The goal potential}

The goal potential must be such that its attraction raises with distance and the force nulls once the goal is reached. One simple potential with these characteristics is the power-law potential,

$$
\psi\left(r_{g}\right)=\left(\frac{r_{g}}{b}\right)^{m}
$$

with the same functional form as used in Refs. 3 and 1 , but here written to be normalized at $r_{g}=b$. Note that $m$ is the potential degree and $b$ the scale length. The force is then

$$
\mathbf{F}_{\psi}\left(r_{g}\right)=-\frac{m}{b}\left(\frac{r_{g}}{b}\right)^{m-1} \hat{\mathbf{e}}_{g},
$$

which has the property $F_{\psi}(0)=0$, which are needed in order for the MO stop at the goal.

\subsection{Minimum distance to an obstacle}

The relative force strength and range between the MO and an obstacle or the goal are determined by the potentials parameters $n, a, m, b$ and the relative distances 
$r_{g}$ and $r_{i}$. The minimum distance the MO will approach an obstacle, given all the potentials parameters above, would be where the obstacle and goal forces balance. To estimate this distance $r_{\min }$, suppose the MO, the obstacle and the goal are colinear and that $r_{g}>r_{i}$. So, the equality $F_{\psi}\left(r_{g}\right)=F_{\varphi}\left(r_{i}\right)$, according to $(3)$ and (5), can be solved to express the radius where the force balance, i.e., the radius of minimum approach

$$
r_{\min }=2 a-a \xi^{\frac{1}{n-1}} \exp \left[-\frac{1}{n} \mathrm{~W}\left(-\frac{n}{n-1} \xi^{\frac{n}{n-1}}\right)\right], \quad \xi \equiv \frac{1}{e} \frac{m}{n} \frac{a}{b}\left(\frac{r_{g}}{b}\right)^{m-1} .
$$

$\mathrm{W}$ is the Lambert Function. ${ }^{10}$ So, with this potential family is possible to analytically calculate the minimum separation between the MO and the obstacles.

\section{Environment Parameters}

Besides the potential analytical properties presented above, it is important to know in how many situations, given a set of parameters, the MO reaches the goal, i.e., the success rate (SR), and how it depends in the overall obstacle configuration in the space.

Two basic parameters indicate how the obstacles populate the space: fulfilling and spacing. The fulfilling is the fraction of the total available space occupied by the obstacles. In a $2 \mathrm{D}$ environment, for example, this would be the fraction of the area covered by the obstacles. The spacing is the mean of the distance of each obstacle and its nearest neighbor, i.e., the mean size of the passages between obstacles.
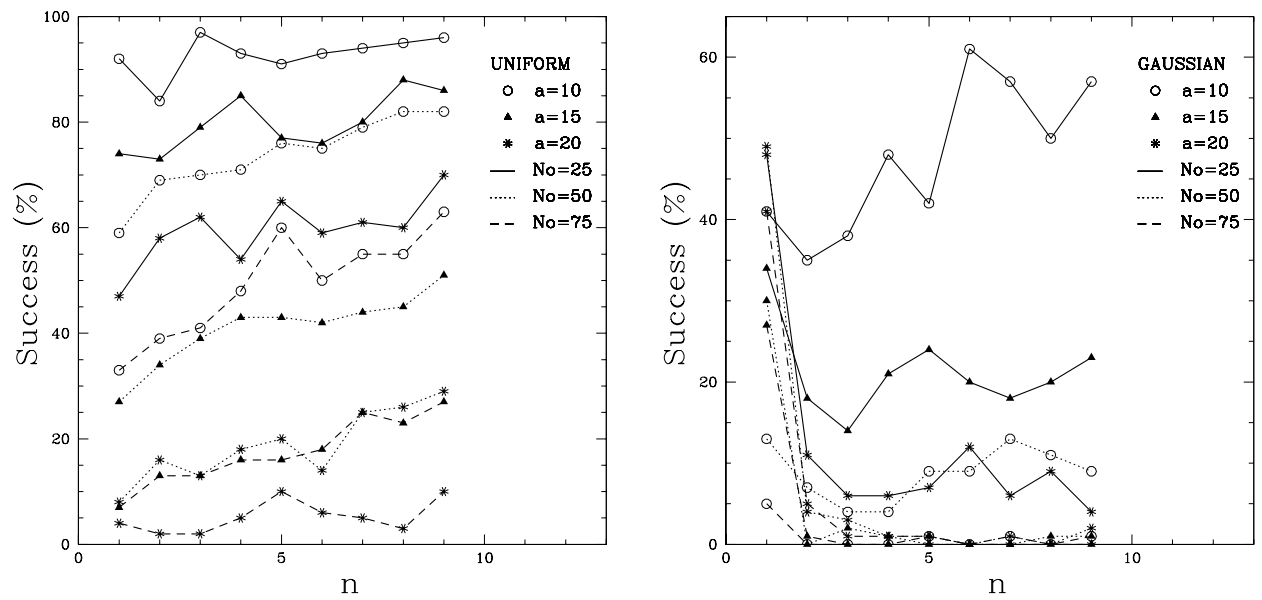

Fig. 2. Mean success rates (SR) as a function of obstacle potential degree $n$, for different obstacles number $N_{0}=25,50,75$ and sizes $a=10,15,20$. Left: uniform obstacle distribution. Right: Gaussian obstacle distribution. Further details in the text. 

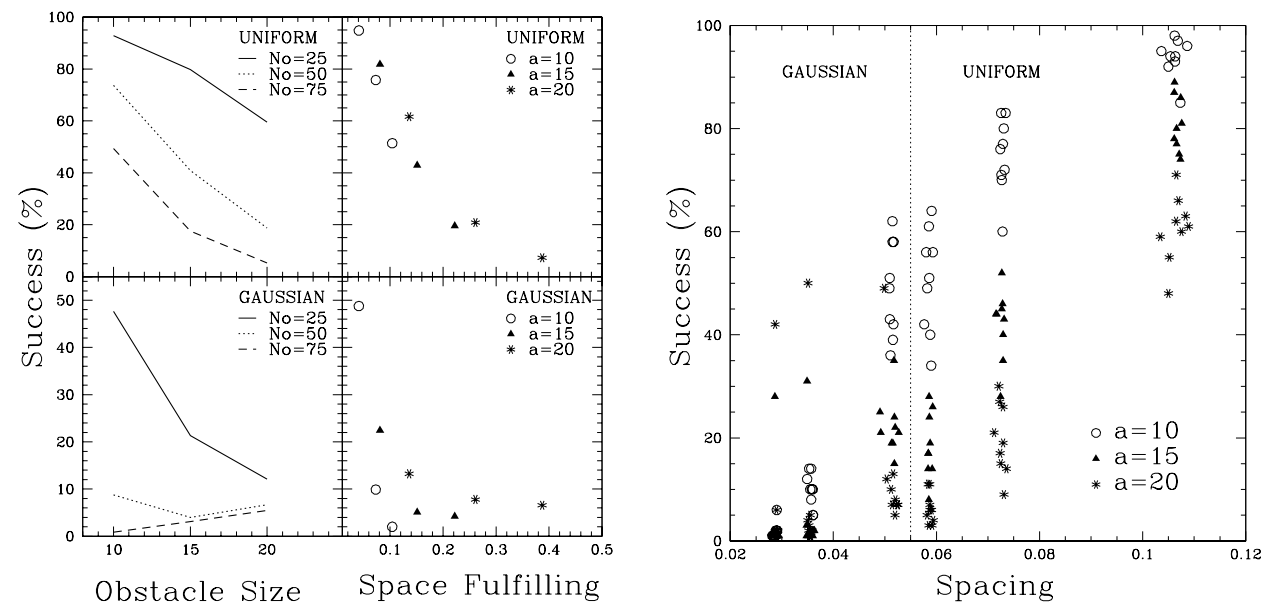

Fig. 3. Left: Success rate as a function of obstacle size (left panels) and space fulfilling (right panels) for the case of uniform distribution (top panels) and Gaussian distribution (bottom panels). In the space fulfilling, for the same $a, N_{o}$ raises rightwards. Right: Success rate as a function of the obstacles spacing, normalized by the space size (DIMx). The part to the left of the dashed line corresponds to the Gaussian obstacles distribution and the part to the right, the uniform distribution.
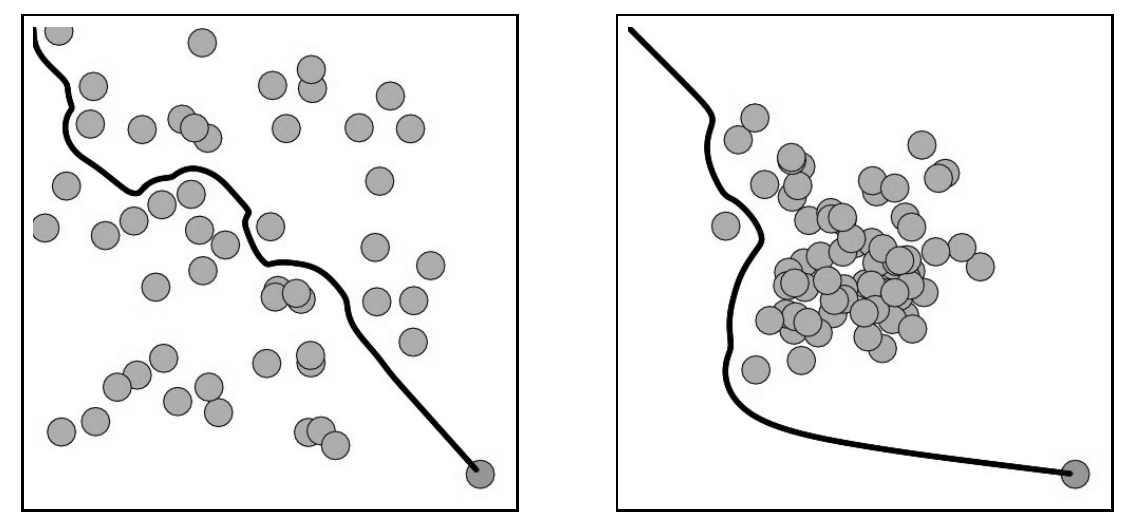

Fig. 4. Two successful cases. Left: uniform distribution, $N o=50, a=15, n=2$. Right: Gaussian distribution, $N o=75, a=15, n=1$.

\section{Simulations}

Simulations were designed in order to evaluate the SR. They were implemented in the Python language in a Linux system. ${ }^{a}$ All simulations consisted of a square 2D space $(\mathrm{DIMx}=\mathrm{DIMy}=500)$ in which a MO starts near the origin towards a goal in the opposite corner (see Figure 4). This was done for the potentials presented here are

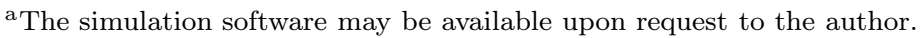


easily expandable for $\mathrm{N}$ dimensional cases. ${ }^{\mathrm{b}}$ All obstacles are circular and their size corresponds to the parameter $a$. The description of non circular obstacles would be a problem of implementing an angular dependence in the potentials, presented here as circularly symmetric. This would greatly complicates the present development without significantly improving the basic ideas and results. The simulations were run for obstacles uniformly distributed in space and for a Gaussian distribution centered at the center of the square. For the Gaussian case, the dispersion was $\sigma=\mathrm{DIMx} / 8$. The individual obstacle coordinates were random. For each case, 100 simulations were run with the same set of parameters, except obstacle locations that were random. In all, $b=120$ and $m=1.8$. The obstacles sizes varied as $a=\{10,15,20\}$, the number of obstacles as $N_{o}=\{25,50,75\}$ and the potential degrees as $n=\{1,2,3,4,5,6,7,8,9\}$, summing up a total of 16200 simulations. The MO velocity is constant and the normalized force was used only to indicate the direction of movement.

\section{Discussion}

The basic idea behind the functional forms of the potentials (2) and (4) is the control of the interaction (force strength and range) between the MO and the obstacles or the goal. This may be done by the parameters $a$ and $n$ : the scale length $a$ specifies the distance where the potential has a certain value (normalized in our case); the potential degree $n$ controls the rate at which the potential changes, i.e., the force strength.

In this way, one may control the distance where the MO will be aware of the obstacles or the goal. For large values of $n$ in the obstacles, the potential will vanish rapidly with distance and the MO will discover the obstacles only when getting too close to them, suffering an abrupt and strong repulsive force. Otherwise, for small values in $n$, the MO will be aware of the obstacles at distances very far from them, since the potential varies slowly with distance. The former situation is adequate for narrow passages between obstacles. The latter situation is more adequate for large scale planning, as the MO has an overall picture of the obstacle distribution in space. The parameter $a$ controls the distance where this interaction with the potential occurs and may be associated with the obstacle size.

In Figure 2 it is shown the SR as a function of the potential degree $n$ and for different obstacle number and sizes. For the uniform distribution of obstacles, the SR raises with $n$, since, the larger the $n$, the closer the MO gets to the obstacles, and thus is able to pass through narrow passages where, for small $n$, it would be trapped in. The case of Gaussian obstacle distribution is distinct in behavior. In general, the lower the $n$, the greater the SR. This is because with small $n$ the MO avoids being trapped in narrow passages (local minima) that are more numerous in a Gaussian distribution. Its sense of the overall obstacle distribution in space forces

\footnotetext{
${ }^{\mathrm{b}}$ In fact, it is just a matter of writing the corresponding Euclidean distances in $r_{i}$ and $r_{g}$.
} 
it to contour the crowd of obstacles in the center (see Figure 4, left.) Figure 4 shows typical successful simulations for both cases.

Figure 3 shows the variation of the SR with obstacle size and space fulfilling, for different obstacle number and sizes. The SR decreases with obstacle sizes (Figure 3, left panels), with gaps of SR between different number of obstacles. Even so, for few large $\left(N_{o}=25, a=20\right)$ or for many small $\left(N_{o}=75, a=10\right)$ the SR is approximately the same, $50-60 \%$.

The SR behavior with space fulfilling is much more uniform. The SR is independent of obstacle number and size individually, but rather a combination of them. In the uniform distribution (top-right), for example, there is not the gap explained above; the points lie in a continuous curve as far as they represent an uniform variation in the space fulfilling. The Gaussian case is not so well behaved, although the points are more concentrated than in the obstacle size graph. In general, the SR varies as a decreasing exponential with the space fulfilling.

When the SR is compared with the obstacle spacing (Figure 3 right), it can be seen that the SR raises with the spacing, since there is local minimum regions where the MO could be trapped in. Besides, the SR raises continually with the spacing, be the obstacle distribution whether uniform of Gaussian.

Also, regarding the GNRON problem, it is possible to solve part of the issue by assigning large values of $n$ to the obstacles near the goal, making these obstacles potential act at short distances and allowing the MO to get close to them in its way toward the goal. The obstacle potential degree could be assigned according to its distance to the goal and to the local space fulfilling.

\section{Conclusions}

In this work, it is proposed a new parameterized potential family for using in path planning algorithms. Both the goal and obstacles potentials have variable scale length and a degree, whose purpose is to provide better control over the path characteristics. By means of these parameters, the minimum distance between the moving object and an obstacle can be controlled; it is also possible to derive an analytical expression to calculate this distance. The success rate of the moving object in reaching the goal raises with the obstacle potential degree $n$ for uniform obstacle distribution and decreases with it in the Gaussian distribution, mainly because of traps between the obstacles.

\section{Acknowledgments}

The author would like to thank the referees, Dr. Edson Prestes, Dr. Leonardo G. Brunnet and Dr. Antônio Kanaan for reading the manuscript and for useful comments.

\section{References}

1. Latombe, J. C. (1991). Robot Motion Planning. Boston: Kluwer Academic Publishers. 
2. Rimon, E., Koditschek, D. E, (1992). Exact robot navigation using artificial potential functions. IEEE Trans. Robot Autom. 8, 501-518.

3. Khatib, O. (1986). Real-time obstacle avoidance for manipulators and mobile robots. Int. J. Robot. Res. 5, 90-98.

4. Lavalle, S. M. (2006). Planning algorithms. Cambridge, MA: Cambridge University Press.

5. Prestes, E., Engel, P. M., Trevisan, M., and Idiart, M. A. (2002). Exploration method using harmonic functions. Robot. Auton. Syst. 40, 25-42.

6. Koren, Y., Borenstein, J. (1991). Potential Field Methods and Their Inherent Limitations for Mobile Robot Navigation. In Proceedings of the IEEE International Conference on Robotics and Automation, Sacramento, California, April 7-12.

7. Ge, S. S., Cui, Y. J. (2000). New Potential Functions for Mobile Robot Path Planning. IEEE Transactions on Robotics and Automation 16, 615-620.

8. Chuang, J.-H., Ahuja, N. (1991). Path Planning Using Newtonian Potential. In Proceedings of the IEEE International Conference on Robotics and Automation, Sacramento, California, April 7-12, 1991.

9. Kim, D. H., Shin, S. (2000). New Repulsive Potential Funcions with Angle Distributions for Local Planning. Advanced Robotics 20, 25-47.

10. Corless, R. M., Gonnet, G. H., Hare, D. E. G., Jeffrey, D. J., Knuth, D. E. (1996). On the Lambert W function. Adv. Comp. Math. 5, 329-359. 